\title{
Alternatively Spliced Variants of the Insulin Receptor Protein Expression in Normal and Diabetic Human Tissues
}

Heike Benecke, Jeffrey S. Flier, and David E. Moller

The Charles A. Dana Research Institute and Harvard-Thorndike Laboratory of Beth Israel Hospital, Department of Medicine, Beth Israel Hospital and Harvard Medical School, Boston, Massachusetts 02215

\begin{abstract}
Two insulin receptor mRNA transcripts resulting from alternative splicing of exon 11 in the receptor gene are expressed in a highly regulated tissue-specific fashion. To date, there is no information about the relative abundance of the protein isoforms encoded by these mRNAs in tissues of normal or diabetic subjects. We employed an antibody raised against the peptide sequence encoded by exon 11 to develop a specific immunoprecipitation assay that is capable of determining the fraction of receptors that include this amino acid sequence. The assay is based on the relative ability of the exon 11 specific monoclonal antibody $(\alpha \operatorname{IR} \alpha)$ compared to a nonspecific anti-receptor antiserum (B-2) to immunoprecipitate solubilized receptors that are first labeled with ${ }^{125} \mathrm{I}$-insulin. The assay was validated using standard curves generated with samples composed of known ratios of the two receptor isoforms. Our results in general confirm observations regarding the relative abundance of the two mRNA species in human tissues, with marked predominance of the exon 11+ isoform in liver, and the exon 11- isoform in leukocytes. Similar amounts of both variants are present in placenta, skeletal muscle, and adipose tissue. In studies with this assay using skeletal muscle extracts from control and noninsulin-dependent diabetes mellitus (NIDDM) subjects, as well as in studies of the two mRNAs in control versus NIDDM muscle using a quantitative polymerase chain reaction assay, we could find no significant difference between control and diabetic subjects. This data contradicts a recent report claiming that normal individuals have only the exon 11- mRNA transcript in their skeletal muscle, whereas NIDDM subjects have similar expression of both mRNAs. Given the emerging evidence that functional differences exist between the two receptor isoforms, these studies are relevant to our understanding of insulin receptor function in health and disease. (J. Clin. Invest. 1992. 89:2066-2070.) Key words: insulin receptor • alternative splicing • non-insulin dependent diabetes mellitus.
\end{abstract}

Address correspondence and reprint requests to David E. Moller, M.D., Division of Endocrinology and Metabolism, SL 436, Beth Israel Hospital, 330 Brookline Ave., Boston, MA 02215.

Received for publication 31 December 1991 and in revised form 6 February 1992.

J. Clin. Invest.

(c) The American Society for Clinical Investigation, Inc. $0021-9738 / 92 / 06 / 2066 / 05 \$ 2.00$

Volume 89, June 1992, 2066-2070

\section{Introduction}

We and others have recently shown that the mRNA that encodes the human insulin receptor is alternatively spliced in a tissue-specific manner (1-3). Thus, a small "cassette" exon (exon 11) encoding 12 amino acids at the carboxy-terminus of the extracellular receptor $\alpha$-subunit is included (Ex11+) or excluded (Ex11-) from the mature mRNA. As examples of this highly regulated process, liver expresses predominantly the Ex11+ transcript, tissues such as cultured dermal fibroblasts and placenta express both mRNAs, and hematopoietic cells express only the Ex11- transcript (1). The potential importance of this finding is underscored by the fact that the varying pattern of tissue-specific mRNA expression initially observed in humans is also precisely conserved in the rat (4). More importantly, emerging evidence indicates that the two encoded receptor isoforms are functionally distinct $(3,5,6)$. Although it is possible that the steady state expression of the two receptor isoforms follows the pattern of expression of the alternatively spliced receptor mRNAs, there is currently no information about the abundance of Ex11- or Ex11+ receptor protein. Indeed, if the two transcripts were differentially translated, or had different half lives, the relative amount of the two proteins might not reflect the ratio of the two transcripts.

Therefore, the primary objective of the current study was to determine the relative abundance of the two receptor protein isoforms in various human tissues. Towards this end, we have developed and applied a novel immunoprecipitation assay that is capable of quantitating the relative abundance of Ex11compared to Ex11+ containing insulin receptors present in small samples of human tissue.

A recent report claimed that an altered ratio of insulin receptor mRNA splice variants exists in skeletal muscle from patients with NIDDM (7). This study concluded that both mRNA transcripts are present in the muscle of diabetic subjects, but that only the Ex11-transcript is present in the muscle of lean control subjects. In order to examine this crucial issue, we have assessed the relative abundance of the two receptor splice variants in skeletal muscle from normal subjects and patients with NIDDM at both the mRNA and protein levels.

\section{Methods}

Materials. Human buffy coat leukocytes were isolated from heparinized whole blood as previously described (1). Human tissue samples of liver and white adipose tissue (omental) were obtained during elective 
surgery from normal subjects. Human placenta was obtained during a cesarian section performed on a normal subject. Samples of skeletal muscle (rectus abdominus) that were obtained from subjects previously characterized as lean normal, obese nondiabetic, and obese non-insulin-dependent diabetes mellitus (NIDDM) ${ }^{1}(8)$ were kindly provided by Dr. Jose F. Caro (East Carolina University School of Medicine, Greenville, NC). Total skeletal muscle (vastus lateralis) RNA samples that were prepared from muscle biopsies obtained from subjects previously characterized as lean normal and NIDDM (9) were kindly provided by Dr. Oluf Pedersen (Steno Diabetes Centre, Gentofte, Denmark) and Dr. Barbara B. Kahn (Beth Israel Hospital, Boston, MA). Informed consent was obtained from all human subjects. Clonal Chinese hamster ovary $(\mathrm{CHO})$ cell lines over-expressing similar numbers of normal human insulin receptors including (CHO-Ex11+) or excluding exon 11 (CHO-Ex11-) were prepared and maintained as previously described (5).

A monoclonal antibody ( $\alpha \mathrm{IR} \alpha)$ was raised against the peptide sequence corresponding to the residues encoded by insulin receptor exon 11. This antibody and the immunizing peptide (CPRKTSSGTGAED) were kindly provided by Dr. Laurel J. Sweet (California Biotechnology Inc., Mountain View, CA). A naturally occurring human IgG anti-insulin receptor antisera (B-2), which we have previously characterized (10), was also employed.

$\left.{ }^{125} \mathrm{I}\right]$ mono-iodinated porcine insulin $(2200 \mathrm{Ci} / \mathrm{mmol})$ was obtained from DuPont-New England Nuclear, Boston, MA. Immobilized protein-A on Trisacryl GF-2000 was obtained from Pierce Chemical Co. Rockford, IL. Thermus aquaticus (Taq) DNA polymerase was obtained from Perkin-Elmer Cetus Instrs., Norwalk, CT. Unless otherwise stated, all other chemicals were obtained from Sigma Chemical Co., St. Louis, MO.

Dual antibody immunoprecipitation assay. Confluent monolayers (10-cm dishes) of transfected CHO cells were solubilized in $5.0 \mathrm{ml}$ of a lysis buffer containing $50 \mathrm{mM}$ Hepes, $1 \%$ Triton X-100, 4 mM EDTA, $0.1 \mathrm{mg} / \mathrm{ml}$ Aprotinin, and $0.35 \mathrm{mg} / \mathrm{ml}$ PMSF. Frozen human tissue samples $(100 \mathrm{mg})$ were pulverized while still frozen, followed by rapid homogenization in $0.5 \mathrm{ml}$ of the lysis buffer above. After $40 \mathrm{~min}$ at $4^{\circ} \mathrm{C}$ (with gentle agitation), insoluble material was removed by centrifugation for $15 \mathrm{~min}$ at $10,000 \mathrm{~g}$.

For samples that were subsequently immunoprecipitated with $\alpha \operatorname{IR} \alpha, 10 \mu \mathrm{l}$ of each cell or tissue lysate containing solubilized insulin receptors was incubated at $4^{\circ} \mathrm{C}$ for $15 \mathrm{~h}$ with $400 \mu \mathrm{l}$ of Krebs-RingersHepes ([KRH] [50 mM Hepes, $\mathrm{pH} 7.6 ; 1.3 \mathrm{mM} \mathrm{MgSO}_{4} ; 1.3 \mathrm{mM}$ $\mathrm{CaCl}_{2} ; 5.1 \mathrm{mM} \mathrm{KCl} ; 130 \mathrm{mM} \mathrm{NaCl}$; and $0.1 \%$ bovine serum albumin]) with $0.1 \mu \mathrm{Ci}{ }^{125} \mathrm{I}$-insulin. After the addition of $1.6 \mu \mathrm{g} \alpha \mathrm{IR} \alpha$ (final dilution 1:200), each sample was incubated at $4^{\circ} \mathrm{C}$ for $3 \mathrm{~h}$. Protein-A Trisacryl suspension $(100 \mu \mathrm{l})$ was then added at a concentration adequate to precipitate all of the IgG. After a 30-min incubation (with gentle agitation) at $4^{\circ} \mathrm{C}$, the immune complex was pelleted by centrifugation in a microfuge. The pellet was washed twice with ice-cold KRH and counted in a gamma counter. Background counts per minute were determined with samples to which $10^{-5} \mathrm{M}$ of cold insulin had been added to the lysate during the binding step.

For samples that were subsequently immunoprecipitated with B-2, an equal volume of cell/tissue lysate was incubated at $4^{\circ} \mathrm{C}$ for $15 \mathrm{~h}$ with $200 \mu \mathrm{l}$ of KRH containing $0.025 \mu \mathrm{Ci}$ of ${ }^{125} \mathrm{I}$-insulin. B-2 antisera was added to a final dilution of $1: 200$ followed by $1 \mathrm{~h}$ incubation at $4^{\circ} \mathrm{C}$. Immunoprecipitates were collected, washed, and quantitated as described above.

Quantitative RNA-based polymerase chain reaction (PCR). Total RNA extracted from human placenta and transfected CHO cells as previously described (1) was used as controls for PCR experiments. Three oligonucleotide primers complementary to sequences flanking

1. Abbreviations used in this paper: $\mathrm{CHO}$, Chinese hamster ovary; NIDDM, non-insulin-dependent diabetes mellitus; PCR, polymerase chain reaction. the segment of the human insulin receptor cDNA corresponding to exon 11 were employed: 5'-TCCTGCAGTTGGACGGTGGTAGACA-3' (A-sense), 5'CCGAATTCGTGGGCACGCTGGTCGA-3' (B-antisense), and 5'-ATCTGCAGAAGCACAACCAGAGTGA-3' (Csense). Specific first-strand cDNA was synthesized using M-MLV reverse transcriptase (Bethesda Research Laboratories, Gaithersburg, MD) with primer B and $5 \mu \mathrm{g}(0.1 \mu \mathrm{g}$ for RNA from CHO cells) of total RNA as previously described (1). Insulin receptor CDNA was amplified in PCR reactions with primer sets $\mathrm{A} / \mathrm{B}$ and $\mathrm{C} / \mathrm{B}$ that included $\left[\alpha-{ }^{32} \mathrm{P}\right]-$ $\operatorname{dCTP}(3,000 \mathrm{Ci} / \mathrm{mmol})$ as described (1). ${ }^{32} \mathrm{P}$-labeled cDNA PCR products were separated by agarose gel electrophoresis followed by quantitation as described (1).

\section{Results}

Validation of a quantitative immunoprecipitation assay. To study the relative expression and tissue distribution of the two alternatively spliced human insulin receptor isoforms at the protein level, we developed a quantitative immunoprecipitation assay using an exon 11-specific antibody $(\alpha \operatorname{IR} \alpha)$ and a nonspecific anti-insulin receptor antisera (B-2). Cell lysates prepared from $\mathrm{CHO}$ transfectants that overexpress exclusively the Ex11- or Ex11+ insulin receptor were used as controls (5). We determined that similar numbers of insulin receptors were expressed by CHO-Ex11- and CHO-Ex11+ clonal cell lines using Scatchard analysis of steady state competitive insulin binding experiments (not shown). Initial experiments revealed that $\alpha \mathrm{IR} \alpha$ specifically immunoprecipitated ${ }^{125} \mathrm{I}$-insulin-labeled receptors from lysates of CHO-Ex $11+$ cells $(357 \pm 6 \mathrm{cpm} / \mu \mathrm{g}$ protein), but did not immunoprecipitate labeled insulin receptors from CHO-Ex 11 - cell lysates $(18.5 \pm 4.7 \mathrm{cpm} / \mu \mathrm{g}$ protein) with similar receptor concentration. The immunoprecipitation of insulin receptors from CHO-Ex $11+$ cell lysates by $\alpha \mathrm{IR} \alpha$ was completely competed by the addition of an excess of the immunizing peptide $(5.5 \pm 1.1 \mathrm{cpm} / \mu \mathrm{g}$ protein). Immunoprecipitation with either antibody was quantitative-a second round of immunoprecipitation yielded less than $10 \%$ additional specific cpm (not shown). Using variable amounts of cell lysates, immunoprecipitation of labeled receptors with each antibody was linear over the range of counts per minute obtained with the subsequent analysis of human tissue samples. The experimental procedure was adjusted to achieve the following controlled conditions: when using the antibody directed against the exon 11 peptide $(\alpha \operatorname{IR} \alpha)$, the number of counts per minute specifically immunoprecipitated from CHO-Ex $11+$ cell lysates was equivalent to the specific counts per minute immunoprecipitated with B-2 from equal volumes of either CHO-Ex11+ or CHO-Ex11- cell lysates.

For each experiment performed with human tissues, a standard curve was generated using samples composed of known varying ratios of CHO-Ex11- and CHO-Ex11+ cell lysates. As shown in Fig. 1, a linear relationship between the relative amount of Ex11+ receptors present in the mixed control cell lysates and the ratio of counts per minute immunoprecipitated with $\alpha \mathrm{IR} \alpha$ vs. B- 2 was observed.

Relative abundance of Ex11- and Ex11+ insulin receptor protein in human tissues. Aliquots of tissue lysate obtained from human buffy coat leukocytes, liver, white adipose tissue, skeletal muscle, and placenta were labeled with ${ }^{125} \mathrm{I}$-insulin and immunoprecipitated separately with $\alpha \mathrm{IR} \alpha$ antibody or B-2 antibody. The ratio of labeled receptors immunoprecipitated with 


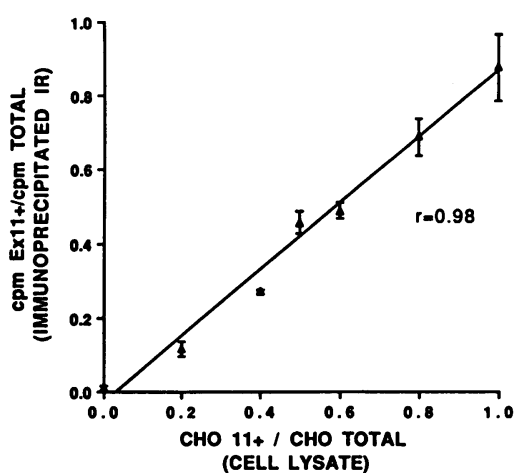

Figure 1. Standard curve for insulin receptor isoform immunoprecipitation. Protein lysates were prepared from $\mathrm{CHO}$ cell transfectants expressing human insulin receptors with (CHO-Ex11+) exon 11 or without (CHOEx11-) exon 11 at similar levels. These two cell lysates were combined together at several fixed ratios followed by labeling with ${ }^{125}$ I-insulin and immunoprecipitation with either an exon 11-specific antibody $(\alpha \operatorname{IR} \alpha)$ or a non-specific anti-insulin receptor antisera (B-2). For each ratio tested, the total (B-2) immunoprecipitated cpm were equivalent (not shown). The relative amount of lysate from CHO-Ex11+ cells (CHO 11+/ CHO Total) and the corresponding ratio of cpm immunoprecipitated with $\alpha \mathrm{IR} \alpha$ (cpm Ex11+)/total cpm immunoprecipitated with B-2 (cpm total) are shown. Each point is the mean ( $($ SEM) of three determinations.

the two antibodies was determined, and the percentage of insulin receptors containing exon 11 was derived from the standard curve generated for each experiment. The relative abundance of Ex11+ receptors differed between the tissues studied. As summarized in Table I, the pattern of tissue-specific protein isoform expression generally correlates with the characteristic tissue-specific pattern of expression for the two receptor mRNA transcripts (1-3). In agreement with the absence of Ex11+ mRNA in hematopoeitic cells, we were not able to detect any Ex $11+$ receptor protein in leukocytes. Given the range of ratios tested in our standard curves, it is still possible that leukocytes do express Ex11+ protein in very small amounts relative to the Ex11- isoform.

In tissues where both receptor variants are expressed, our ability to accurately quantitate the relative abundance of the two isoforms is complicated by the small difference in insulin binding affinity $(\operatorname{Ex} 11->\operatorname{Ex} 11+)$ that is known to exist $(3,5)$. Since receptors were labeled by binding to ${ }^{125} \mathrm{I}$-insulin, it is possible that our determination of the relative amount of Ex $11+$ receptors was slightly lower than the actual abundance.

Table I. Relative Abundance of Alternatively Spliced Insulin Receptor Protein and mRNA Isoforms in Human Tissues

\begin{tabular}{lcc}
\hline \multicolumn{1}{c}{ Tissue } & \% Ex11+ Protein $^{*}$ & \% Ex11+ mRNA \\
\hline Liver & $75.8 \pm 12.6$ & $85-90^{\ddagger}$ \\
Skeletal muscle & $45.2 \pm 3.4^{8}$ & $78^{11}$ \\
Leukocyte & 0 & $0^{\ddagger}$ \\
Adipose tissue & $62 \pm 22$ & $28-70^{\ddagger}$ \\
Placenta & $39 \pm 13.2$ & $45-50^{\ddagger}$
\end{tabular}

* The percentage of Ex11+ containing insulin receptors was quantitated using the dual antibody immunoassay described in the present study. Unless otherwise noted, each value is the mean of three experiments, each performed in triplicate. ${ }^{*}$ The percentage of Ex11+ insulin receptor mRNA is derived from the range of data reported by Moller et al. (1), and Seino and Bell (2). ${ }^{8}$ Mean of 15 subjects; see Fig. 3. "Mean of 11 subjects in present study; see Fig. 2 and Results.
In a tissue such as muscle, where we calculate a value of $\sim 50 \%$ Ex 11 + receptors, the actual value would correct to $67 \%$ assuming a twofold difference in steady state tracer binding affinity. This factor may therefore account for the modest discrepancy between relative mRNA and protein expression that we have observed in tissues such as muscle or liver. Another factor that could contribute to this discrepancy in muscle is the fact that vastus lateralis was used for RNA studies, whereas rectus abdominus was used for studies of receptor protein.

Insulin receptor isoform expression in skeletal muscle from NIDDM subjects. The relative expression of insulin receptor splice variants in skeletal muscle was determined at both the RNA and protein levels. Total RNA extracted from vastus lateralis skeletal muscle biopsies from lean normal $(n=7)$ and $\operatorname{NIDDM}(n=5)$ subjects was reverse-transcribed followed by PCR-amplification of CDNA with two primer sets flanking exon $11(\mathrm{~A} / \mathrm{B}, \mathrm{C} / \mathrm{B})$. As shown in the example presented in Fig. 2 , PCR with either primer set from all RNA samples resulted in two specific bands corresponding to the presence ( $721 \mathrm{bp}$ with $\mathrm{A} / \mathrm{B}, 300 \mathrm{bp}$ with $\mathrm{C} / \mathrm{B}$ ) or absence of exon 11 (685 bp with A/B, $264 \mathrm{bp}$ with $\mathrm{C} / \mathrm{B})$. Quantitation of the relative amount of ${ }^{32} \mathrm{P}$ labeled PCR product corresponding to the Ex11+ vs. Ex11transcripts was expressed as the percentage of Ex $11+$ PCR product vs. the total. We have previously validated these methods by demonstrating accurate quantitation of steady state mRNA levels using standard curves composed of known mixed amounts of the two transcripts present in total RNA from CHO cell transfectants (1). With primer set A/B the mean $( \pm$ SEM) percentage of Ex $11+$ mRNA was $77.7 \pm 8.8$ for control samples compared with $78.7 \pm 5.6$ for NIDDM samples. With primer set $C / B$ the values were $75.9 \pm 6.6$ and $76.9 \pm 6.7$ for controls and NIDDM subjects, respectively. Thus, no difference in the relative abundance of the two $\mathrm{mRNA}$ transcripts was apparent in skeletal muscle RNA from control versus NIDDM subjects.

The relative abundance of insulin receptor protein isoforms was determined with a separate group of rectus abdominus skeletal muscle samples from subjects well characterized as lean normal, obese non-diabetic and obese NIDDM $(n=5$ each). Tissue lysates were prepared from each muscle sample and assayed in multiple experiments using the immunoprecipitation assay described above. The total amounts of ${ }^{125} \mathrm{I}$-labeled insulin receptors immunoprecipitated with B-2 antibody

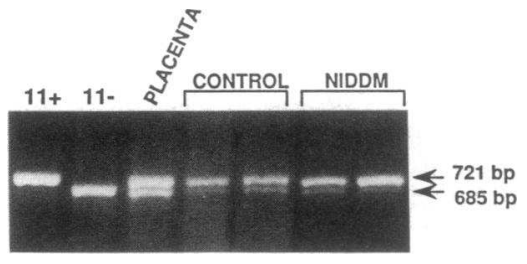

Figure 2. PCR amplifcation of alternatively spliced insulin receptor mRNA transcripts. In this example, total cellular RNA from CHO cell transfectants expressing human insulin receptors with exon 11

$(11+)$ or without (11-) exon 11, human placenta, and skeletal muscle (vastus lateralis) from two control subjects and two NIDDM subjects was reverse-transcribed with a specific oligonucleotide primer (primer B, see Methods) followed by PCR amplification of first-strand cDNA with one of two primer sets used (primers A and B, see Methods). This ethidium bromide stained $1.7 \%$ agarose gel reveals the expected specific 721 and 685 bp PCR product DNA fragments corresponding to the presence or absence, respectively, of exon 11 in the starting mRNA. For muscle from each of the four subjects shown here, both PCR products were visible on the original gel. 


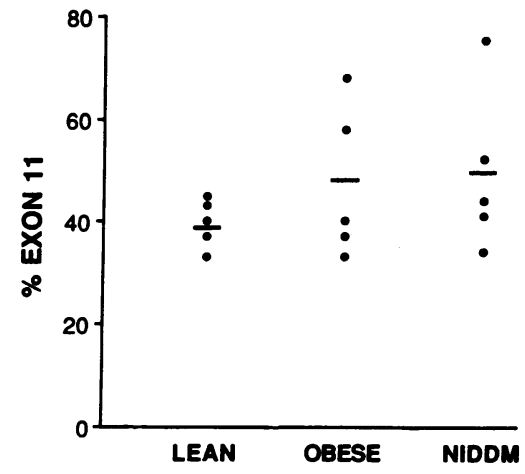

within $10 \mu \mathrm{l}$ of tissue lysate were labeled with ${ }^{125}$ I-insulin followed by immunoprecipitation with either an exon 11-specific antibody $(\alpha \operatorname{IR} \alpha)$ or a non-specific anti-insulin receptor antisera (B-2). Results are expressed as the percentage of insulin receptors containing exon 11 (percentage of exon 11), which is derived from the ratio of $\mathrm{cpm}$ immunoprecipitated with $\alpha \mathrm{IR} \alpha / \mathrm{cpm}$ immunoprecipitated with B-2. Each point represents the mean value for a single subject measured in three or more experiments (each in triplicate). The mean for each group of subjects is shown as a horizontal bar.

yielded results that are consistent with the data published by Caro et al. (11) in a study of very similar patients. A modest decrease in insulin receptor number in skeletal muscle in subjects with obesity and NIDDM was observed. The mean value for lysates from normal controls was $4,821 \pm 492 \mathrm{cpm} / \mathrm{mg}$ protein versus $3,725 \pm 301$ from obese nondiabetic subjects, and $3,089 \pm 120$ from NIDDM subjects. The relative abundance of protein isoforms was expressed as the percentage of Ex11+ receptors precipitated versus the total. Mean values for each subject are shown in Fig. 3. The mean of the percentage of Ex11+ in lysates from lean normals was $39 \pm 5$ compared to $47 \pm 6$ for obese nondiabetics, and $49 \pm 7$ for obese NIDDM subjects.

\section{Discussion}

Over the past 20 years, investigators have studied the role of the insulin receptor in mediating the important actions of insulin. Recently, it has become apparent that two receptor mRNA transcripts (containing or excluding exon 11) are expressed at characteristic tissue-specific ratios as a result of alternative splicing (1-3). To date, a limited functional assessment of the two encoded insulin receptor isoforms has been performed through parallel studies of transfected cell lines expressing either cDNA species $(3,5,6,12)$. These studies reveal that although both receptor isoforms are active as tyrosine kinases and have the ability to signal various intracellular pathways (5, 12 ), the two receptors differ functionally in at least two ways. Thus, there are significant differences in ligand binding affinity $(3,5,12)$ and the internalization kinetics of the two species differ as well $(5,6)$.

In this report, we have provided the first information on the relative distribution of the two receptor species in human tissues. Using appropriately controlled conditions and standard curves based on varying known ratios of the two isoforms, we have developed an immunoprecipitation assay that is an accurate means for determining the relative abundance of Ex11vs. Ex $11+$ insulin receptors in small amounts of human tissue
( $\leq 1-2 \mathrm{mg}$ ). The results indicate that the tissue-specific pattern of receptor protein isoform expression generally correlates well with the previously described pattern of mRNA transcript expression (1-3). Thus, as predicted by the absence of Ex11+ mRNA in leukocytes, we detected no Ex11+ protein in this tissue. Similarly, the predominance of the Ex11+ mRNA in liver is reflected in the assessment of protein abundance.

It is not known whether the two receptor proteins can be packaged as hybrid heterodimers or exist only as homodimers. In a situation where the steady state levels of the two mRNAs are equal, assuming that each transcript is translated with equal efficiency and that the turnover rate of the two proteins is comparable, random sorting of half-receptors would be expected to result in a higher proportion of holoreceptors containing at least one Ex1 1 + half-receptor. Using our current approach, we were not able to address these important issues. Additional studies will be required to directly assess the translation of the two transcripts, the turnover rate of the two proteins and the potential for receptor hybrid formation.

It is intriguing to speculate that changes in the relative expression of the two insulin receptor isoforms could somehow contribute to in vivo insulin resistance. Apart from several mutations in the insulin receptor gene which we and other groups have identified in patients with severe insulin resistance (13), little is known about the molecular basis for observed decreases in receptor number (14) and impairment of insulin-stimulated receptor autophosphorylation and/or tyrosine kinase activity (15) in tissues derived from human subjects with common insulin resistant states (obesity and NIDDM). Based on current knowledge, it seems unlikely that variation in the primary structure of the insulin receptor is common in patients with such disorders $(16,17)$. Using a PCR approach, Mosthaf et al. have recently reported that skeletal muscle RNA from normal nondiabetic subjects contains only the Ex11- mRNA transcript, whereas muscle RNA samples from NIDDM subjects contain both mRNA species (7). This finding is at odds with our previous assessment of the abundance of the two mRNAs in skeletal muscle from two normal subjects (1), and with a prior report by Mosthaf et al., where two PCR products corresponding to the presence of both mRNAs in muscle from a normal subject are depicted (3).

We have now employed an RNA-based radiolabeled PCR product assay which we previously validated using standard curves to be quantitative over a range of known ratios of the two mRNA transcripts (1). Using two PCR primer sets, our current results again indicate that both mRNA transcripts are present (with Ex11+ predominating) in skeletal muscle (vastus lateralis) obtained from a group of nondiabetic and NIDDM subjects. Moreover, the results with both primer sets agree, and we can detect no significant differences between NIDDM and control subjects. As a completely independent experimental approach to this issue, we analyzed the relative abundance of the two protein isoforms in skeletal muscle samples from another anatomic location (rectus abdominus), obtained from a different patient population. It is clear from our results that both receptor isoforms are expressed in the skeletal muscle of lean normals, obese nondiabetics, and obese diabetics. In addition, we were not able to detect significant differences in the relative abundance of receptor isoforms between the three groups of subjects tested. Further studies using a larger number of tissue samples will be needed to determine whether the slightly higher mean percentage of Ex11+ receptors in obese 
nondiabetics and obese NIDDM evident in Fig. 3 represents a significant quantitative difference between these groups.

There are several potential explanations for the discrepancy between our findings and those reported by Mosthaf et al. First, the source and condition of skeletal muscle differed in the two studies; we used biopsied vastus lateralis and rectus abdominus, whereas they studied samples of gastrocnemius obtained after limb amputation for vascular disease (7). Second, their results are based on the use of a single PCR oligonucleotide primer set, and no standard curve with samples of varying known composition was reported to validate their PCR approach. It is possible that small differences between their control and NIDDM samples could have been markedly accentuated over 40 cycles of PCR by a difference in the efficiency with which each of the two competing cDNA templates were amplified. An internal standard curve is essential to rule out such a possibility.

In summary, several aspects of this study are worthy of note. First, using a new immunoprecipitation assay, we have provided the first available information, in any species, on the relative steady state expression of the two known protein isoforms of the insulin receptor in a variety of tissues (see note added in proof below). At first approximation, these data indicate that the tissue expression of the two proteins parallels earlier observations at the mRNA level. This is most obvious in tissues that display extreme variations at the mRNA level (e.g., liver and leukocytes), but also holds true in tissues with reasonable amounts of both transcripts, such as muscle and fat. Second, these data controvert the recent provocative observation of Mosthaf et al. (7) who noted a marked disparity between normal and NIDDM subjects with respect to the abundance of the two mRNA transcripts in muscle. Specifically, we have been unable to distinguish between controls and diabetics with respect to Ex11+ receptor abundance at the mRNA level, which they assessed, or at the protein level. At this time, it cannot be answered whether the difference between these two studies represents heterogeneity of patient populations, the source and condition of muscle preparations, or the methodologies employed. Based on our data, we are confident that both normal and NIDDM subjects express similar amounts of the two receptor isoforms in the muscles that we have examined. Future investigation will be required to identify potential subtle quantitative differences that might exist. Given the fact that the two receptor isoforms are functionally distinct, further pursuit of this issue may be warranted.

Note added in proof: Since the submission of this paper, a study by Sesti et al. (Diabetes. 41:6-11. 1992) was published that used human polyclonal anti-insulin receptor IgG to demonstrate that Ex11+ is the predominant receptor isoform present in human liver.

\section{Acknowledgments}

We are indebted to Laurel J. Sweet and her colleagues at California Biotechnology, Inc. (Mountain View, CA) for providing the $\alpha \mathrm{IR} \alpha$ antibody and immunizing peptide. This study would not have been possible without the generous help of Jose F. Caro (E. Carolina University School of Medicine), Oluf Pedersen (Steno Diabetes Centre, Gentofte Denmark) and Barbara B. Kahn (Beth Israel Hospital, Boston, MA) who provided valuable human samples. We also wish to thank Morris White (Joslin Diabetes Center, Boston, MA) for providing CHO Ex11- cells and Jonathan Whittaker (State University of New York, Stonybrook) for providing a human insulin receptor cDNA used in the preparation of CHO Ex11+ cells. Technical advice from Ken Siddle (University of Cambridge, United Kingdom) is greatly appreciated. This work was supported by a Physician Scientist Award to Dr. David E. Moller from the National Institute on Aging, a grant from the Juvenile Diabetes Foundation, also to Dr. Moller, a fellowship from the Deutsche Forschungsgemeinschaft (Be 1243-1) to Dr. Heike Benecke, and National Institute of Health grant NIDDK 28082-10 to Dr. Jeffrey S. Flier.

\section{References}

1. Moller, D. E., A. Yokota, J. F. Caro, and J. S. Flier. 1989. Tissue-specific expression of two alternatively spliced insulin receptor mRNA's in man. $\mathrm{Mol}$. Endocrinol. 3:1263-1269.

2. Seino, S., and G. I. Bell. 1989. Alternative splicing of human insulin receptor messenger RNA. Biochem. Biophys. Res. Commun. 159:312-316.

3. Mosthaf, L., K. Grako, T. J. Dull, L. Coussens, A. Ullrich, and D. A. McClain. 1990. Functionally distinct insulin receptors generated by tissue-specific alternative splicing. EMBO (Eur. Mol. Biol. Organ.) J. 9:2409-2413.

4. Goldstein, B. J., and A. L. Dudley. 1990. The rat insulin receptor: primary structure and conservation of tissue-specific alternative messenger RNA splicing. Mol. Endocrinol. 4:235-244.

5. Yamaguchi, Y., J. S. Flier, A. Yokota, H. Benecke, J. M. Backer, and D. E. Moller. 1991. Functional properties of two naturally occurring isoforms of the human insulin receptor in Chinese hamster ovary cells. Endocrinology. 129:2058-2066.

6. Vogt, B., J. M. Carrascosa, B. Ermel, A. Ullich, and H.-U. Haring. 1991. The two isotypes of the human insulin receptor (HIR-A and HIR-B) follow different internalization kinetics. Biochem. Biophys. Res. Comm. 177:1013-1018.

7. Mosthaf, L., B. Vogt, H. Haring, and A. Ullrich. 1991. Altered expression of insulin receptor types A and B in the skeletal muscle of non-insulin-dependent diabetes mellitus patients. Proc. Natl. Acad. Sci. USA 88:4728-4730.

8. Dohm, G. L., E. B. Tapscott, W. J. Pories, D. J. Dabbs, E. G. Flickinger, D. Meelheim, T. Fushiki, S. M. Atkinson, C. W. Elton, and J. F. Caro. 1988. An in vitro human muscle preparation suitable for metabolic studies: decreased insulin stimulation of glucose transport in muscle from morbidly obese and diabetic subjects. J. Clin. Invest. 82:486-494.

9. Pedersen, O., J. F. Bak, P. H. Anderson, S. Lund, D. E. Moller, J. S. Flier, and B. B. Kahn. 1990. Evidence against altered expression of Glut 1 or Glut 4 in skeletal muscle of patients with obesity or non-insulin-dependent diabetes mellitus. Diabetes. 39:865-870.

10. Flier, J. S., C. R. Kahn, D. B. Jarrett, and J. Roth. 1976. Characterization of antibodies to the insulin receptor: a cause of insulin-resistant diabetes in man. J. Clin. Invest. 58:1442-1449.

11. Caro, J. F., M. K. Sinha, S. M. Raju, O. Ittoop, W. J. Pories, E. G. Flickinger, D. Meelheim, and G. L. Dohm. 1987. Insulin receptor kinase in human skeletal muscle from obese subjects with and without non-insulin dependent diabetes. J. Clin. Invest. 79:1330-1337.

12. McClain, D. A. 1991. Different ligand affinities of the two human insulin receptor splice variants are reflected in parallel changes in sensitivity for insulin action. Mol. Endocrinol. 5:734-739.

13. Moller, D. E., and J. S. Flier. 1991. Insulin resistance: mechanisms, syndromes, and implications. N. Engl. J. Med. 325:938-948.

14. Olefsky, J. M. 1981. Lilly Lecture 1980 . Insulin resistance and insulin action: an in vitro and in vivo perspective. Diabetes. 30:148-161.

15. Haring, H., and B. Obermaier-Kusser. 1989. Insulin receptor kinase defects in insulin-resistant tissues and their role in the pathogenesis of NIDDM. Diabetes Metab. Rev. 5:431-441.

16. O'Rahilly, S., W. H. Choi, P. Patel, R. C. Turner, J. S. Flier, and D. E. Moller. 1991. Detection of mutations in the insulin receptor gene in non-insulin dependent diabetic patients by analysis of single-stranded conformation polymorphisms. Diabetes. 40:777-782.

17. Kusari, J., U. S. Verma, J. B. Buse, R. R. Henry, and J. M. Olefsky. 1991. Analysis of the gene sequences of the insulin receptor and the insulin-sensitive glucose transporter (GLUT-4) in patients with common-type non-insulin dependent diabetes mellitus. J. Clin. Invest. 88:1323-1330. 